\title{
Roughness and Reynolds Number Effects on the Flow Past a Rough-to-Smooth Step Change
}

\author{
Amirreza Rouhi, Daniel Chung and Nicholas Hutchins
}

\begin{abstract}
We report direct numerical simulations (DNSs) of open-channel flow with a step change from three-dimensional sinusoidal rough surface to smooth surface. We investigate the persistence of non-equilibrium behaviour beyond this step change (i.e. departures from the equilibrium smooth openchannel flow) and how this depends on 1) roughness virtual origin $\epsilon / h$ ? (scaled by the channel height $h), 2$ ) roughness size $k / h$ ?, 3) roughness shape? and 4 ) Reynolds number $R e_{\tau}$ ? To study (1), the roughness origin was placed aligned with, below (step-up) and above (step-down) the smooth patch. To study (2), the equivalent sand-grain roughness of the aligned case was decreased from $k_{s}^{+} \simeq 160$ to 106 . To study (3) and (4) the step-down case at $R e_{\tau} \simeq 395$ was compared with a backward-facing step case at $R e_{\tau} \simeq 527$, and DNS of square rib rough-to-smooth case at $R e_{\tau} \simeq 1160$ (Ismail et al., J. Fluid Mech., vol. 843, 2018, pp. 419-449). Results showed that $\epsilon / h$ affects the departure from equilibrium by a large extent, while $k / h$, roughness shape and $R e_{\tau}$ have a marginal influence. The departure from equilibrium was found to be related to the near-wall amplification of Reynolds shear stress, which in turn depends on $\epsilon / h$, i.e. higher $\epsilon / h$ leads to higher amplification.
\end{abstract}

\section{Introduction}

Changes in surface roughness occur in many fabricated or natural applications, e.g. the edges of forests or the bio-fouled patches of a ship hull. Here, we investigate the surface change in the streamwise direction from a rough patch to a smooth patch, collectively noted as rough-to-smooth surface change. The surface change causes various phenomena, two of which are mostly addressed

Amirreza Rouhi*, Daniel Chung, Nicholas Hutchins

Department of Mechanical Engineering, University of Melbourne, Victoria, Australia, e-mail: amirreza.rouhi@unimelb.edu.au 
in the literature: 1) departure from equilibrium, and 2) formation of an internal boundary layer (IBL) [1] (Fig. 1 $1 a$ ). The IBL is a layer influenced by the new surface condition. The lower part of the IBL which is in equilibrium with the new surface is termed the internal equilibrium layer (IEL). Once the IEL meets the boundary-layer edge, a new equilibrium is reached.
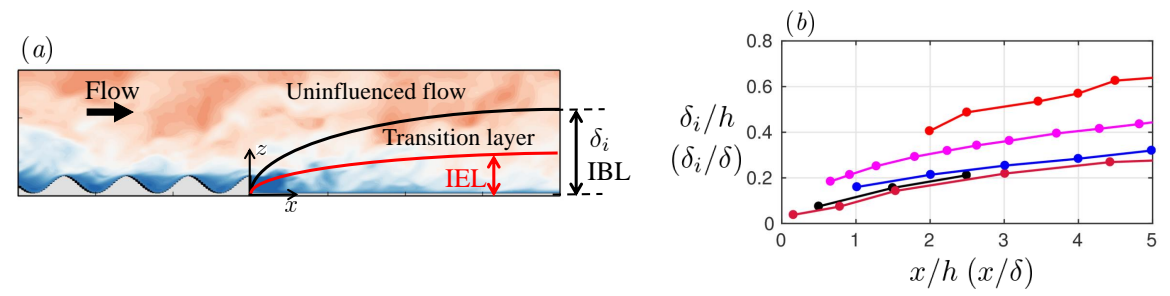

Fig. 1 (a) Growth of the internal boundary layer (IBL) and equilibrium layer (IEL) past a rough-to-smooth step change. (b) Compilation of the IBL thicknesses $\delta_{i}$ from studies in table 1 . For boundary layer studies, $x$ and $\delta_{i}$ are scaled by the boundary layer thickness $\delta$; for channel flow (open-channel) scaling is with the channel halfheight (height) $h$. The line colors are consistent with the colors of studies in Table 1

Table 1 A collection of previous rough-to-smooth studies that performed experiments in a boundary layer (first three) or DNS in a channel flow (last two).

\begin{tabular}{|c|c|c|c|c|c|}
\hline Study & $\begin{array}{c}\delta_{i} \\
\text { definition }\end{array}$ & $R e_{\tau}$ & $\begin{array}{l}\text { Roughness } \\
\text { type }\end{array}$ & $\begin{array}{c}\text { Roughness } \\
\text { virtual origin } \epsilon\end{array}$ & $\begin{array}{l}\text { Roughness } \\
\text { size } k\end{array}$ \\
\hline $\begin{array}{l}\text { Antonia \& } \\
\text { Luxton } 3\end{array}$ & $z^{1 / 2}$ slope 2 & 970 & $2 \mathrm{D}$ bars & $\begin{array}{r}\epsilon<0 \\
\stackrel{\longrightarrow}{\longrightarrow}\end{array}$ & 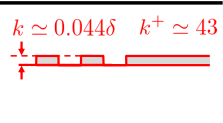 \\
\hline $\begin{array}{c}\text { Carper } \\
\text { Porté-Agel [5] }\end{array}$ & $0.99 U_{\text {rough }} 4$ & 8800 & wire mesh & $\begin{array}{r}\epsilon \simeq 0 \\
-\infty \cos a\end{array}$ & $\begin{array}{l}k \simeq 0.008 \delta \quad k^{+} \simeq 70 \\
\end{array}$ \\
\hline $\begin{array}{c}\text { Hanson \& } \\
\text { Ganapathisubramani 6] }\end{array}$ & $z^{1 / 2}$ slope 2 & 1800 & grit & 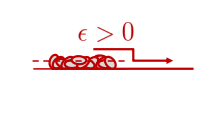 & $\begin{array}{l}k \simeq 0.034 \delta \quad k^{+} \simeq 61 \\
\frac{1}{4}\end{array}$ \\
\hline Ismail et al. 7 & $0.99 U_{\text {rough }} 4$ & 1160 & $2 \mathrm{D}$ bars & 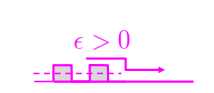 & $\begin{array}{l}k \simeq 0.083 h \quad k^{+} \simeq 96 \\
\frac{+}{4} \square\end{array}$ \\
\hline Rouhi et al. 8 & log slope 9 & 430 & egg carton & 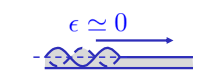 & $\begin{array}{l}k \simeq 0.056 h \quad k^{+} \simeq 39 \\
\text { 战 }\end{array}$ \\
\hline
\end{tabular}

Table 1 lists some of the previous rough-to-smooth studies, and Fig. 1 $(b)$ compiles their reported IBL thicknesses $\delta_{i} / h\left(\delta_{i} / \delta\right)$ relative to the channel height $h$, or boundary layer thickness $\delta$. Considering Fig. 1 ( $b$ ), a large scatter is seen in the results. The potential causes are listed in table 1 including different 1) flow configurations, i.e. boundary layer versus channel flow, 2) 
$\delta_{i}$ definitions [8], 3) Reynolds numbers, 4) Roughness types, 5) Roughness (virtual) origins $\epsilon / h$ and 6 ) Roughness sizes $k / h$. Here, we investigate the

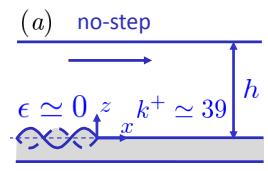

(e) backward-facing step

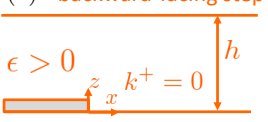

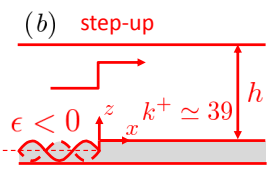
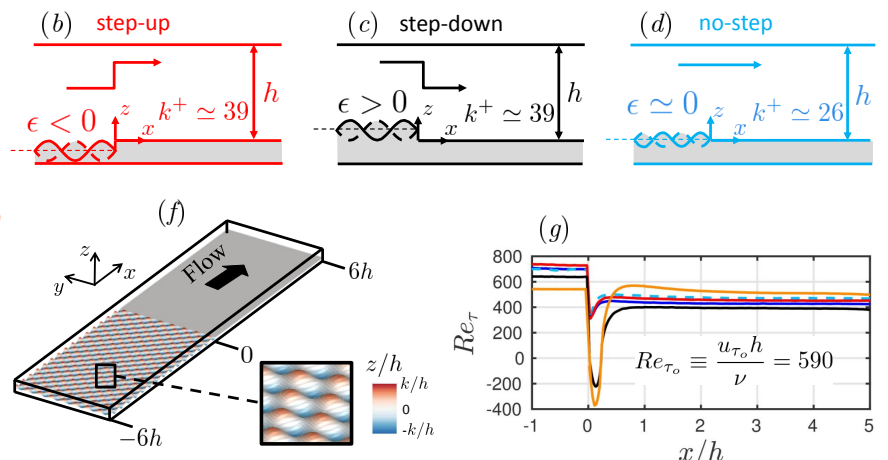

Fig. $2(a-d)$ Simulation cases: no-step (-), step-up ( $(-)$, step-down $(-)$, nostep (---) and backward-facing step (- $)$. $(f)$ computational domain for no-step case $(-)$ and $(g)$ local Reynolds number $R e_{\tau} \equiv u_{\tau} h / \nu$ for all cases.

role of (3) to (6). In particular we investigate how much the departure from the expected equilibrium (equilibrium smooth open-channel flow) is affected by: 1) $\epsilon / h$ ? 2) $k / h$ ? 3) roughness shape? and 4) Reynolds number? To answer (1), we consider the no-step case (- ), Fig. 22(a), with roughness size $k / h=$ 0.056 as the sinusoidal roughness amplitude, and recess (Fig. $2 p$ ) and elevate (Fig. 2 $c$ ) the upstream rough patch by $k / h$ to create a step-up (-) and a step-down $(-)$ configuration. To answer (2), we decrease the roughness size of the no-step case to $k / h=0.037\left(---, k_{s}^{+}=106\right.$, Fig. 2 $\left.2 d\right)$. To answer (3) and (4) the step-down case is compared with a backward-facing step simulation (- , Fig. 2ee), and DNS of Ismail et al. 7] (- ).

\section{Direct Numerical Simulation}

We used a validated fourth-order code [10]. The domain is open channel (Fig. 2e), periodic in the streamwise $(x)$ and spanwise $(y)$ directions. Freeslip condition is imposed at the top, and no-slip condition is imposed at the bottom surface via an immersed boundary method [11. The $x z$-origin is placed at the step change. The domain length is $12 h$, equally divided between the rough and smooth patches. With this length, the flow within the IBL is almost insensitive to the patch length and domain periodicity [8]. The flow was driven by the global $R e_{\tau_{o}} \equiv u_{\tau_{o}} h / \nu=590$ based on $u_{\tau_{o}}$ averaged over time and the entire bottom surface. However, local $R e_{\tau} \equiv u_{\tau} h / \nu$ varies from $650-700$ over the rough patch to $400-450$ over the smooth patch (Fig. 2 g). 


\section{Results}
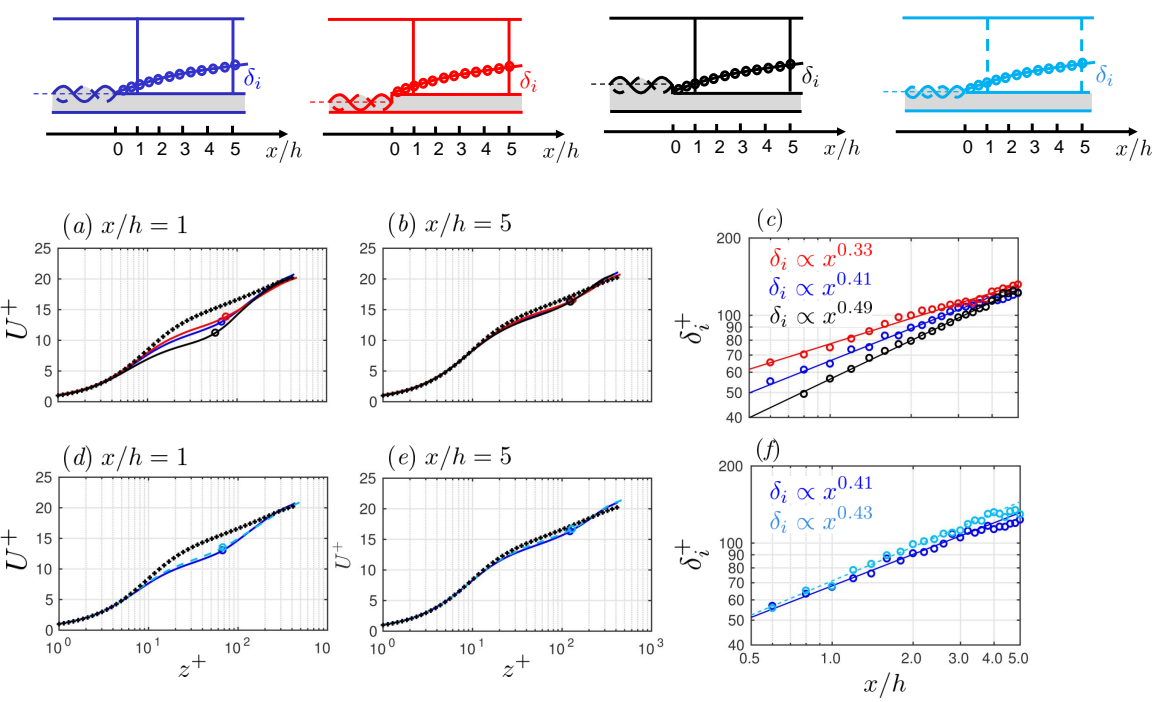

Fig. 3 Roughness origin effect on $(a, b) U^{+}$profiles and (c) IBL thicknesses $\delta_{i}^{+}$. Roughness size effect on $(d, e) U^{+}$profiles and $(f) \delta_{i}^{+} . \delta_{i}^{+}$is also marked on the profiles. The quantities in plus units are normalized by the local $u_{\tau}$ and $\nu$. Fully developed open-channel flow at $R e_{\tau}=437(+)$.

The effect of roughness origin is studied in Fig. 3 $3(a-c)$ by comparing the no-step case ( - ) with step-up ( -$)$ and step-down ( - ) cases. Roughness size effect is studied in Fig. 3 $3(d-f)$ by comparing the two no-step cases with different $k / h(-, k / h=0.056,---, k / h=0.037)$. To study the departure from the expected equilibrium, we compare the mean velocity $U^{+}$profiles with the fully developed open-channel profile at $R e_{\tau}=437$, the expected equilibrium flow in the far downstream. The departure appears as a downshift in the $U^{+}$profile which at $x / h=1$ (Fig. 3a,d) penetrates down to the viscous sublayer. We also quantify $\delta_{i}$ using Elliott's [9] definition which we previously found to be more consistent with the IBL concept $[8]$. Considering the roughness origin effect (Fig. $3 a-c$ ), initially at $x / h=1$ (Fig. $3 a$ ), the stepdown case yields the largest downshift in $U^{+}$. Consequently, at $x / h=1, \delta_{i}^{+}$is the lowest for the step-down case (Fig. $3 c$ ). Nevertheless, further downstream the roughness origin effect gradually diminishes. By $x / h=5$ (Fig. $3 b$ ) the $U^{+}$profiles and $\delta_{i}^{+}$values (Fig. $3 c$ ) are almost the same as each other. This implies the faster recovery in the step-down case, also seen in the faster $\delta_{i}^{+}$ growth rate. On the other hand, considering the roughness size effect (Fig. $3 d-$ $f$ ), we observed a much smaller influence than the roughness origin, seen both in the very similar $U^{+}$(Fig. $3 d, e$ ) and $\delta_{i}^{+}$profiles (Fig. 3f f). 

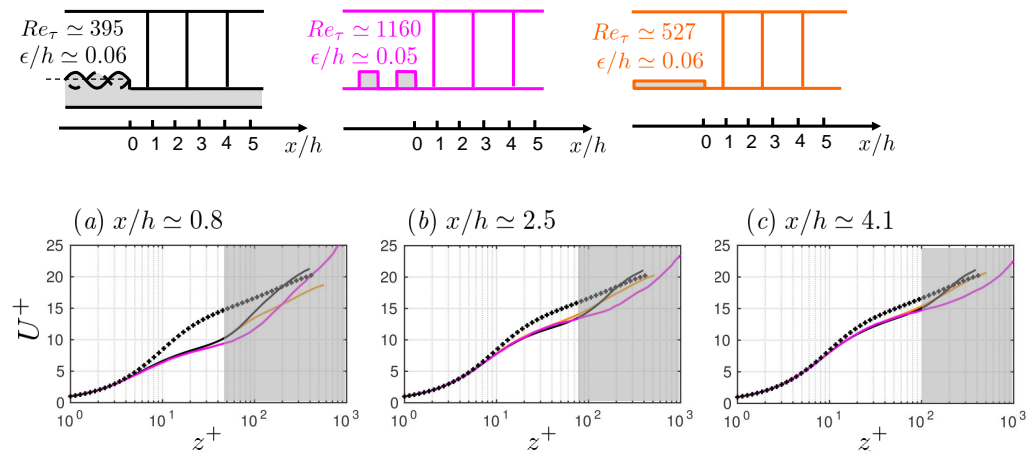

Fig. 4 Comparison of the $U^{+}$profiles between the step-down case ( - ), DNS of Ismail et al. [7. (- , fourth case in Table 1) and a backward-facing step flow (). The reported $R e_{\tau}$ is its recovered value over the smooth surface. The unshaded areas highlight the same levels of downshift for the three datasets.

In Fig. 4 4 we show that $\epsilon / h$ also has a larger influence than $R e_{\tau}$ or roughness shape. We compare three cases with almost equal $\epsilon / h$, but different $R e_{\tau}$ and upstream surface types: 1$)$ step-down case $(-$, Fig. 2a $), 2)$ Ismail et al. 7 ] $\left(-\right.$, Table 1) and 3) backward-facing step case $(-$, Fig. $2 f)$. The $U^{+}$ profiles in Fig. 4 $(a-c)$ yield the same levels of downshift for all three cases.
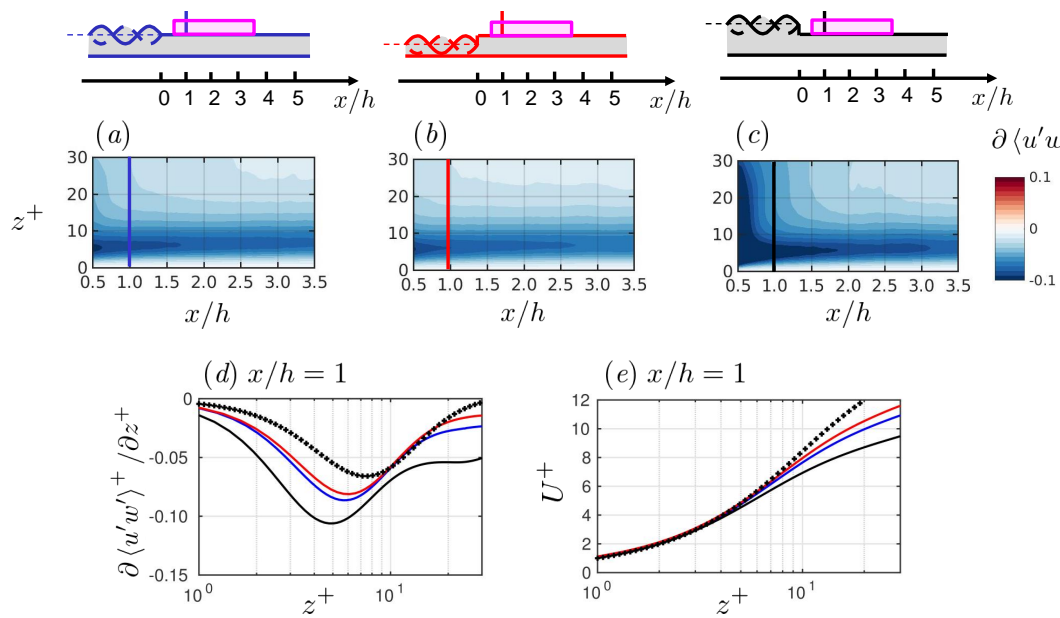

Fig. 5 Roughness origin effect on the Reynolds shear-stress gradient $\partial\left\langle u^{\prime} w^{\prime}\right\rangle^{+} / \partial z^{+}$. $(a, b, c) 2 D$ fields of $\partial\left\langle u^{\prime} w^{\prime}\right\rangle^{+} / \partial z^{+}$near the wall for $z^{+} \leq 30$, highlighted with magenta frames in the top domains. Profiles of $(d) \partial\left\langle u^{\prime} w^{\prime}\right\rangle^{+} / \partial z^{+}$and $(e) U^{+}$at $x / h=1$, indicated in the domains and $2 D$ fields at the top.

We attempted to understand the underlying mechanism (e.g. advection, pressure gradient, Reynolds stresses) behind the observed downshift in $U^{+}$ 
profiles, and its connection with $\epsilon / h$. We studied the momentum budgets down to $z^{+} \simeq 30$ (not shown), where the departure from the equilibrium profile starts. Results showed that below $z^{+} \simeq 30$ the momentum balance is reduced to $\partial\left\langle u^{\prime} w^{\prime}\right\rangle^{+} / \partial z^{+}=\partial^{2} U^{+} / \partial z^{+^{2}}$, where $\left\langle u^{\prime} w^{\prime}\right\rangle^{+}$is the Reynolds shear-stress. In other words, $U^{+}$up to the buffer region merely depends on $\left\langle u^{\prime} w^{\prime}\right\rangle^{+}$. For the rough-to-smooth cases, $\left\langle u^{\prime} w^{\prime}\right\rangle^{+} / \partial z^{+}$yields a stronger inner peak that is closer to the wall than the equilibrium counterpart (Fig. 5d). This causes a thinning of the viscous sublayer and downshift in $U^{+}$(Fig. 5e). For the step-down case this inner peak is strongest and penetrates deeper to the viscous sublayer, causing the maximum departure from equilibrium.

\section{Conclusions}

We performed DNSs of rough-to-smooth step change to determine the degree to which the departure from equilibrium conditions beyond the step-change depends on 1) roughness origin $\epsilon / h$ ?, 2) roughness size $k / h$ ?, 3) roughness shape? and 4) Reynolds number $R e_{\tau}$ ? To answer (1) the roughness origin was placed aligned with (no-step), below (step-up) and above (step-down) the smooth patch. To answer (2) the roughness size of the no-step case was decreased from $k_{s}^{+} \simeq 160$ to $k_{s}^{+} \simeq 106$. To answer (3) and (4) the step-down case was compared with a backward-facing step simulation and DNS of [7]. Results showed that $\epsilon / h$ affects the departure from equilibrium by a large extent, while $k / h$ has a marginal influence. Same conclusion was drawn from comparing the internal layer thicknesses. Study of (3) and (4) showed that the departure from equilibrium is mainly influenced by $\epsilon / h$ rather than $R e_{\tau}$ or

roughness shape. The departure from equilibrium can be primarily attributed to the near-wall amplification of Reynolds shear-stress which in turn depends on $\epsilon / h$.

\section{References}

1. S.A. Savelyev, P.A. Taylor, Boundary Layer Meteorol. 115, 1 (2005)

2. R.A. Antonia, R.E. Luxton, J. Fluid Mech. 48, 721 (1971)

3. R.A. Antonia, R.E. Luxton, J. Fluid Mech. 53, 737 (1972)

4. W. Pendergrass, S.P.S. Arya, Atmos. Environ. 18, 1267 (1984)

5. M.A. Carper, F. Porté-Agel, Boundary Layer Meteorol. 126, 157 (2008)

6. R.E. Hanson, B. Ganapathisubramani, J. Fluid Mech. 795, 494 (2016)

7. U. Ismail, T.A. Zaki, P.A. Durbin, J. Fluid Mech. 843, 419 (2018)

8. A. Rouhi, D. Chung, N. Hutchins, J. Fluid Mech. (accepted)

9. W.P. Elliott, Trans. Am. Geophys. Union 39, 1048 (1958)

10. D. Chung, J.P. Monty, A. Ooi, J. Fluid Mech. 742, R3 (2014)

11. A. Scotti, Phys. Fluids 18, 031701 (2006) 


\section{University Library}

\section{- M M I N E R VA \\ A gateway to Melbourne's research publications}

Minerva Access is the Institutional Repository of The University of Melbourne

Author/s:

Rouhi, A;Chung, D;Hutchins, N

Title:

Roughness and Reynolds Number Effects on the Flow Past a Rough-to-Smooth Step Change

Date:

2019

\section{Citation:}

Rouhi, A., Chung, D. \& Hutchins, N. (2019). Roughness and Reynolds Number Effects on the Flow Past a Rough-to-Smooth Step Change. Orlu, R (Ed.) Talamelli, A (Ed.) Peinke, $J$ (Ed.) Oberlack, M (Ed.) iTi 2018: Progress in Turbulence VIII, 226, pp.81-86. Springer International Publishing. https://doi.org/10.1007/978-3-030-22196-6_13.

Persistent Link:

http://hdl.handle.net/11343/253283 